\title{
Article
}

\author{
Doi 10.5943/sif/6/1/30
}

\section{Growth promoting effect of microbial inoculants on Acacia nilotica grown under nursery conditions}

\section{Dash S and Gupta N}

Plant Pathology and Microbiology Division, Regional Plant Resource Centre, Bhubaneswar - 751015 Odisha, India

Dash S, Gupta N 2021 - Growth promoting effect of microbial inoculants on Acacia nilotica grown under nursery conditions. Studies in Fungi 6(1), 400-407, Doi 10.5943/sif/6/1/30

\begin{abstract}
Present study was carried out on Acacia nilotica seedlings grown under different bioinoculation treatments by using laboratory grown liquid cultures of 10 fungal isolates, 11 Rhizobium isolates and 5 other bacterial isolates. Microbial cultures were screened for their effects on growth of Acacia nilotica grown in greenhouse conditions. The selected microbial inoculants were assessed as considering their individual performance or in combination with the other inoculants in specific ratios. The higher performance was shown with the combination of Penicillum chrysogenum Thom. 1, B 5 bacterial isolate, R10 and /or R11 isolates of Rhizobium. Further, it was observed that the above microbial mixed culture inoculation was promoted plant growth specifically number of leaves and the leaf biomass. Therefore, nursery application of mixed microbial inoculants, specifically as above could be beneficial for the production of quality planting material (QPM) of Acacia nilotica and subsequent field applications.
\end{abstract}

Keywords - Acacia nilotica - Aspergillus - Growth - Penicillium - Phosphate solubilization Rhizobium

\section{Introduction}

Nitrogen $(\mathrm{N})$, phosphorus $(\mathrm{P})$ and potassium $(\mathrm{K})$ can be considered as the major plant nutrients which occupy a key place in balanced use of fertilizer in plant growth. Phosphorus is important nutrient for growth of legumes and the process of atmospheric nitrogen fixation (Huda et al. 2007). Microorganisms are capable of mineralizing and solubilizing bound nutrients in soil, and make nutrients available in rhizosphere or inhabitant host, thereby enhances the plant growth (Dabas \& Kaushik 1998, Sahgal et al. 2004, Tilak et al. 2005, Gupta et al. 2007, Hameeda et al. 2008). It is known that a large number of seedlings of forest species should be used in afforestation in dry deciduous conditions. Microbial application at the nursery stage was also found to be useful in enhancing productivity in some forest trees like Albizzia, Acacia Dalbergia etc. (Varma \& Mathur 1989, Sahgal et al. 2004, Dash et al. 2013).

Acacia nilotica is an indigenous multipurpose tree, widely planted under various agroforestry systems. This can be an early successional plant in nutrient poor soils (Purohit et al. 2007). Leaves and pods of Acacia nilotica have been used to feed the goat as traditional food in some parts of the world (Talpur et al. 2008). Faye et al. (2008), studied the symbiotic relationships with specific Rhizobium spp. of two protected forests of Acacia nilotica var. tomentosa in the Senegal river valley. Sarr \& Lesueur (2007), studied the influence of soil fertility on the rhizobial competitiveness for nodulation of A. nilotica in nursery conditions and reported that the interactions of inoculated Rhizobium spp. host plant genotype and soil type mainly determine the competitiveness, nodulation, and symbiotic nitrogen fixation. Sharma et al. (2001) studied the 
ectomycorrhizal dependency and growth responses of A nilotica and Albizzia lebbeck seedlings with the inoculation of specific densities of spores of Pisolithus tinctorius.

Therefore, knowing the importance of planting Acacia nilotica in the afforestation programme in the nutrient deficient soil, the present study was carried out to develop and appropriate use of bioinoculants for nursery applications. Furthermore, to enhance the quality of planting materials are used in transplantation and field application.

\section{Materials \& methods}

Different species of microbial inoculants isolated from

The experiment was carried out in the greenhouse condition (temperature of $35 \pm 2^{\circ} \mathrm{C}$ and $80 \pm 5 \%$ relative humidity) within polybags of $2.5 \mathrm{~kg}$ capacity filled with sandy loam soil of 6.27 . Six phosphate solublizing fungi, Penicillium griseofulvum Diercks (PF1), Penicillium restrictum Gilman \& Abott (PF2), Penicillium chrysogenum Thom. 1 (PF 3), Aspergillus sp.1 (PF4), Aspergillus ornatus (PF5), Aspergillus wentii (PF6), four iron leaching fungi Penicillum expansum (IF1), Paecilomyces variotii (IF2), Cunnighamella elegans (IF3), Penicillium chrysogenum Thom 2 (IF3), five phosphate solublizing bacteria (PB) and 11 Rhizobium isolates were used for the inoculation studies.

\section{Screening of microbial cultures for growth of Acacia nilotica}

Liquid culture of fungi ( 7 days old, Czapek dox medium, $4.5 \mathrm{pH}$ ) and bacteria ( 5 days old, Nutrient broth, $\mathrm{pH}$ 7.2) were added to each pot $(25 \mathrm{ml} / \mathrm{pot})$ prior to seed sowing. The experiment was set in 20 replications. Finally, observations were recorded of 120 days plants (after seed sowing) for shoot height, number of leaves, leaflets, branches developed, fresh and dry biomass of leaves and stems.

\section{Dual inoculation experiment}

In second phase of experiment, two best microbial strains were selected for tree legume on the basis of their performances in previous experiment. Following experimental sets were taken into consideration; Control: without any inoculation (T1), 50ml broth of Penicillium chrysogenum (T2), 50ml broth of phosphate solubilising bacteria PB 5 (T3), $50 \mathrm{ml}$ broth of both fungus and bacteria used in T1 and T2 [T4], 100ml broth of $P$. chrysogenum $+50 \mathrm{ml}$ of PB 5 [T5], $50 \mathrm{ml}$ broth of $P$. chrysogenum $+100 \mathrm{ml}$ of PB 5 [T6].

\section{Dual Inoculation with Rhizobium isolates}

In third phase of experiment, two best microbial strains were selected for tree legume on the basis of their performances in previous experiment [1] control uninoculated [2], inoculated with Penicillium chrysogenum [3], inoculated with PB5 [4], individual inoculation of Rhizobium (11 isolates) [5], inoculation with Penicillium chrysogenum, individual Rhizobium [6], inoculation with PB 5 with individual Rhizobium.

\section{Growth analysis}

Growth parameters were recorded for the experimental plants such as shoot height $(\mathrm{cm})$, root length $(\mathrm{cm})$, number of leaves, leaflets branches, seedling height $(\mathrm{cm})$, collar diameter $(\mathrm{mm})$, fresh and ry biomass of leaf, stem, root, shoot seedling (g) (Al-Garni 2006, Sah et al. 1998, Tewari et al. 2006). One-way ANOVA was done by following Sokal \& Rohlf (1981).

\section{Results}

\section{Effect of microbial inoculation on Acacia nilotica}

The plants of $A$. nilotica grown under different treatment along with control untreated plants 
exhibited good growth in terms of plant height, biomass. In spite of this, significant variations were not observed among all the treatments affect plant growth performances. However, fungal strain PF 3 and bacterial isolate PB5 showed better effects in enhancing plant growth as compared to other microbial inoculants. Bacterial strains showed good effects in enhancing biomass of leaves as compared to fungal inoculants. Finally, these PF $3+$ PB 5 were selected for further experimentations on dual inoculations (Table 1). In primary screening, it was observed that in case of root length best results was given by the treatment PF $3(33.34 \pm 3.54 \mathrm{~cm})$ followed by PF6 $(29.76 \pm 2.99 \mathrm{~cm})$ and PF4 $(29.60 \pm 2.52 \mathrm{~cm})$. Shoot height was enhanced by PF3 $(137.58 \pm 8.59$ $\mathrm{cm})$ and PF2 $(133.56 \pm 13.05 \mathrm{~cm})$. Inoculation of PB5 and PF 6 were produced high fresh biomass. In case of dry biomass of leaves highest value was found in PB5 (4.13 $\pm 0.93 \mathrm{~g})$ and PF 3 (3.15 \pm $0.36 \mathrm{~g})$. Hence, screening experiment exhibited the potential of PF3 + PB5 inoculations and selected for further studies.

\section{Effect of dual inoculation on Acacia nilotica}

The plants of $A$. nilotica grown under different treatment along with control untreated plants exhibited good growth. Fungal strain PF3+ PB5 showed better effects in enhancing plant growth as compared to other treatments in combination (Table 2). In the secondary screening shoot height was enhanced in the treatment of PF3 + PB5 in 1:1 ratio $(140.54 \pm 5.88 \mathrm{~g})$. This combination had also improved the biomass of plants. The combination of both the microbes in equal proportion was found to be the most effective for the plant.

\section{Effect of dual inoculations with Rhizobium}

The plants of $A$. nilotica grown under different treatment along with control untreated plants exhibited good growth in terms of plant height and biomass. Microbial strain PF 3 + PB5 showed better effects in enhancing plant growth as compare to other treatments in combination with R4 and R5 respectively (Tables 3-6). In the present study, individual inoculation of R4 exhibited increase of shoot height $(118.72 \pm 6.58 \mathrm{~cm})$ compared to B5 + R5 $(111.36 \pm 9.59 \mathrm{~cm})$. Root length was found to be enhanced in case of PF3+R4 $(44.48 \pm 2.30 \mathrm{~cm}), \mathrm{PF} 3+\mathrm{B} 5+\mathrm{R} 10(42.06 \pm 1.93 \mathrm{~cm})$ followed by treatment of PF3 + PB5 + R11 $(40.00 \pm 6.64 \mathrm{~cm})$. The plants which were grown under this microbial combination of inoculants had produced good biomass, collar diameter as compared to other treatments. By analyzing all the growth parameters for Acacia nilotica, the best treatments were combination of PF3 (Penicillium chrysogenum Thom 1), PB5 and R11 (Rhizobium sp.). Though the test plants were found to be infected with Rhizobium but a very poor performance was observed as far as root nodule formation is concerned.

Table 1 Growth performance of Acacia nilotica grown under different microbial treatments $(\mathrm{p}>0.05 *, \mathrm{p}>0.01 * *)$

\begin{tabular}{lllllll}
\hline Growth & \multicolumn{5}{c}{ Treatments } \\
\cline { 2 - 7 } Parameters & $\mathbf{1}$ & $\mathbf{2}$ & $\mathbf{3}$ & $\mathbf{4}$ & $\mathbf{5}$ & $\mathbf{6}$ \\
\hline Root length & $31.24 \pm$ & $35.00 \pm$ & $44.74 \pm$ & $39.80 \pm$ & $44.78 \pm$ & $42.56 \pm$ \\
(cm) & 10.27 & 3.78 & $6.66^{*}$ & 2.94 & $11.93^{*}$ & 7.42 \\
Shoot height & $84.46 \pm$ & $132.92 \pm$ & $134.56 \pm$ & $140.54 \pm$ & $117.78 \pm$ & $124.06 \pm$ \\
(cm) & 25.00 & $8.87^{* *}$ & $6.74^{* *}$ & $5.88^{* *}$ & $12.72^{*}$ & $21.24^{* *}$ \\
Fresh biomass & $4.33 \pm$ & $7.62 \pm$ & $13.21 \pm$ & $14.69 \pm$ & $13.70 \pm$ & $14.57 \pm$ \\
(g) & 2.57 & 1.45 & $3.22^{* *}$ & $3.58^{* *}$ & $3.46^{* *}$ & $1.57^{* *}$ \\
Dry biomass & $1.82 \pm$ & $5.04 \pm$ & $6.41 \pm$ & $7.55 \pm$ & $7.56 \pm$ & $7.98 \pm$ \\
(g) & 1.42 & 1.17 & $1.64^{* *}$ & $1.76^{* *}$ & $1.85^{* *}$ & $0.55^{* *}$ \\
\hline
\end{tabular}

1- control, 2- Penicillium chrysogenum Thom. 1,3-Bacterial isolate 5, 4 -both fungal and bacterial strains equal amount, 5- both fungal and bacterial isolates in 2:1 ratio, 6- both fungal and bacterial isolates in 1:2 ratio, $(\mathrm{p}>0.05 *, \mathrm{p}>0.01 * *)$ 
Table 2 Effect of selected bioinoculant fungal and bacterial strains on growth of Acacia nilotica grown under pot culture conditions

\begin{tabular}{llll}
\hline & Shoot height $\mathbf{( c m )}$ & Fresh biomass leaves $(\mathbf{g})$ & Dry biomass leaves $(\mathbf{g})$ \\
\hline CONTROL & $112.76 \pm 29.60$ & $3.54 \pm 1.93$ & $2.07 \pm 0.77$ \\
Penicillium grisefulvum & $114.02 \pm 14.81$ & $4.23 \pm 1.08$ & $2.14 \pm 1.02$ \\
Penicillium restrictum & $133.56 \pm 13.05$ & $4.41 \pm 1.39$ & $1.90 \pm 0.87$ \\
Penicillium crysogenum & $137.58 \pm 8.59^{*}$ & $7.25 \pm 2.02$ & $3.15 \pm 0.36^{*}$ \\
Aspergillus species & $116.30 \pm 22.95$ & $4.13 \pm 2.64$ & $0.85 \pm 0.55$ \\
Aspergillus ornatus & $119.42 \pm 20.45$ & $11.76 \pm 4.94$ & $1.80 \pm 0.11$ \\
Aspergillus wentii & $122.72 \pm 17.30$ & $1.42 \pm 0.57$ & $4.57 \pm 2.87 *$ \\
Penicillum expansum & $122.98 \pm 8.34$ & $3.26 \pm 1.27$ & $1.08 \pm 0.41$ \\
Paecilomyces variotii & $121.70 \pm 13.22$ & $9.82 \pm 6.02$ & $1.35 \pm 0.34$ \\
Cunnighamella elegans & $97.22 \pm 29.73$ & $7.94 \pm 1.97$ & $1.94 \pm 0.80$ \\
Bacterial isolate PB1 & $115.76 \pm 12.70$ & $8.13 \pm 2.07$ & $3.04 \pm 1.36$ \\
Bacterial isolate PB2 & $104.96 \pm 6.92$ & $8.27 \pm 4.91$ & $2.88 \pm 1.04$ \\
Bacterial isolate PB3 & $108.26 \pm 10.07$ & $8.27 \pm 4.91$ & $3.02 \pm 2.04$ \\
Bacterial isolate PB4 & $119.96 \pm 7.41$ & $9.89 \pm 1.99$ & $2.96 \pm 0.20$ \\
Bacterial isolate PB5 & $95.18 \pm 33.11$ & $12.89 \pm 2.75^{*}$ & $4.13 \pm 0.93^{*}$ \\
\hline
\end{tabular}

Table 3 Effects of selected microbial inoculants and Rhizobium on growth of Acacia nilotica

\begin{tabular}{|c|c|c|c|c|c|c|c|c|}
\hline Treatment & $\begin{array}{l}\text { Shoot } \\
\text { height } \\
\text { (cm) }\end{array}$ & $\begin{array}{l}\text { Root } \\
\text { length } \\
(\mathrm{cm}) \\
\end{array}$ & $\begin{array}{l}\text { Seedling } \\
\text { height } \\
(\mathrm{cm})\end{array}$ & $\begin{array}{l}\text { FBM } \\
\text { leaf } \\
(\mathrm{g})\end{array}$ & $\begin{array}{l}\text { DBM } \\
\text { leaf } \\
(\mathrm{g}) \\
\end{array}$ & $\begin{array}{l}\text { Collar } \\
\text { dia } \\
(\mathrm{cm})\end{array}$ & $\begin{array}{l}\text { FBM } \\
\text { root } \\
(\mathrm{g}) \\
\end{array}$ & $\begin{array}{l}\text { DBM } \\
\text { root } \\
(\mathrm{g}) \\
\end{array}$ \\
\hline C 1 & $\begin{array}{l}70.94 \pm \\
6.62\end{array}$ & $\begin{array}{l}19.58 \pm \\
1.44\end{array}$ & $\begin{array}{l}90.52 \pm \\
7.44\end{array}$ & $\begin{array}{l}8.14 \pm \\
1.31\end{array}$ & $\begin{array}{l}3.79 \pm \\
0.67\end{array}$ & $\begin{array}{l}18.60 \pm \\
2.70\end{array}$ & $\begin{array}{l}2.75 \pm \\
0.18\end{array}$ & $\begin{array}{l}1.47 \pm \\
0.13\end{array}$ \\
\hline $\mathrm{C2}$ & $\begin{array}{l}95.90 \pm \\
23.30 * *\end{array}$ & $\begin{array}{l}30.96 \pm \\
3.31 * *\end{array}$ & $\begin{array}{l}126.84 \pm \\
24.30^{* *}\end{array}$ & $\begin{array}{l}10.27 \pm \\
1.50\end{array}$ & $\begin{array}{l}5.29 \pm \\
0.67\end{array}$ & $\begin{array}{l}21.20 \pm \\
1.92\end{array}$ & $\begin{array}{l}2.62 \pm \\
0.41\end{array}$ & $\begin{array}{l}1.37 \pm \\
0.29\end{array}$ \\
\hline C3 & $\begin{array}{l}84.66 \pm \\
10.49\end{array}$ & $\begin{array}{l}28.10 \pm \\
6.20 *\end{array}$ & $\begin{array}{l}112.76 \pm \\
14.56^{*}\end{array}$ & $\begin{array}{l}10.33 \pm \\
0.58\end{array}$ & $\begin{array}{l}6.37 \pm \\
0.25^{*}\end{array}$ & $\begin{array}{l}20.22 \pm \\
2.12\end{array}$ & $\begin{array}{l}2.49 \pm \\
0.25\end{array}$ & $\begin{array}{l}1.37 \pm \\
0.21\end{array}$ \\
\hline R1 & $\begin{array}{l}83.94 \pm \\
5.33\end{array}$ & $\begin{array}{l}22.48 \pm \\
1.34\end{array}$ & $\begin{array}{l}106.42 \pm \\
6.57\end{array}$ & $\begin{array}{l}9.46 \pm \\
3.40\end{array}$ & $\begin{array}{l}5.37 \pm \\
2.46\end{array}$ & $\begin{array}{l}21.40 \pm \\
1.52\end{array}$ & $\begin{array}{l}2.49 \pm \\
0.43\end{array}$ & $\begin{array}{l}1.37 \pm \\
0.25\end{array}$ \\
\hline $\mathbf{R} 2$ & $\begin{array}{l}108.02 \pm \\
9.29 * *\end{array}$ & $\begin{array}{l}24.26 \pm \\
6.78\end{array}$ & $\begin{array}{l}132.28 \pm \\
12.90^{* *}\end{array}$ & $\begin{array}{l}10.32 \pm \\
1.39\end{array}$ & $\begin{array}{l}6.59 \pm \\
0.48^{*}\end{array}$ & $\begin{array}{l}21.60 \pm \\
1.95\end{array}$ & $\begin{array}{l}2.76 \pm \\
0.26\end{array}$ & $\begin{array}{l}1.47 \pm \\
0.17\end{array}$ \\
\hline $\mathbf{R 3}$ & $\begin{array}{l}99.04 \pm \\
9.43 * *\end{array}$ & $\begin{array}{l}30.10 \pm \\
10.27 * *\end{array}$ & $\begin{array}{l}129.14 \pm \\
17.55^{* *}\end{array}$ & $\begin{array}{l}10.60 \pm \\
1.18\end{array}$ & $\begin{array}{l}5.24 \pm \\
0.98\end{array}$ & $\begin{array}{l}20.60 \pm \\
0.89\end{array}$ & $\begin{array}{l}2.80 \pm \\
0.49\end{array}$ & $\begin{array}{l}1.60 \pm \\
0.33\end{array}$ \\
\hline R4 & $\begin{array}{l}118.72 \pm \\
6.58 * *\end{array}$ & $\begin{array}{l}20.28 \pm \\
1.51\end{array}$ & $\begin{array}{l}139.00 \pm \\
6.90 * *\end{array}$ & $\begin{array}{l}9.84 \pm \\
1.60\end{array}$ & $\begin{array}{l}6.00 \pm \\
1.41\end{array}$ & $\begin{array}{l}20.60 \pm \\
0.89\end{array}$ & $\begin{array}{l}3.11 \pm \\
0.38\end{array}$ & $\begin{array}{l}1.69 \pm \\
0.18\end{array}$ \\
\hline R5 & $\begin{array}{l}79.14 \pm \\
11.16\end{array}$ & $\begin{array}{l}25.24 \pm \\
4.02\end{array}$ & $\begin{array}{l}104.38 \pm \\
7.44\end{array}$ & $\begin{array}{l}10.73 \pm \\
1.24\end{array}$ & $\begin{array}{l}6.22 \pm \\
1.07\end{array}$ & $\begin{array}{l}22.00 \pm \\
2.35\end{array}$ & $\begin{array}{l}2.67 \pm \\
0.36\end{array}$ & $\begin{array}{l}1.79 \pm \\
0.47\end{array}$ \\
\hline R6 & $\begin{array}{l}71.90 \pm \\
7.74\end{array}$ & $\begin{array}{l}31.12 \pm \\
5.84 * *\end{array}$ & $\begin{array}{l}103.02 \pm \\
4.24\end{array}$ & $\begin{array}{l}11.45 \pm \\
1.80 * *\end{array}$ & $\begin{array}{l}6.14 \pm \\
1.05\end{array}$ & $\begin{array}{l}19.00 \pm \\
2.55\end{array}$ & $\begin{array}{l}3.15 \pm \\
0.33\end{array}$ & $\begin{array}{l}1.74 \pm \\
0.45\end{array}$ \\
\hline R7 & $\begin{array}{l}67.82 \pm \\
9.69\end{array}$ & $\begin{array}{l}24.94 \pm \\
7.27\end{array}$ & $\begin{array}{l}92.76 \pm \\
12.03\end{array}$ & $\begin{array}{l}11.28 \pm \\
1.65 * *\end{array}$ & $\begin{array}{l}7.10 \pm \\
1.04 * *\end{array}$ & $\begin{array}{l}21.20 \pm \\
3.56\end{array}$ & $\begin{array}{l}1.93 \pm \\
0.30\end{array}$ & $\begin{array}{l}1.01 \pm \\
0.29\end{array}$ \\
\hline R8 & $\begin{array}{l}65.46 \pm \\
9.69\end{array}$ & $\begin{array}{l}28.24 \pm \\
3.32\end{array}$ & $\begin{array}{l}93.70 \pm \\
10.35\end{array}$ & $\begin{array}{l}9.14 \pm \\
1.45\end{array}$ & $\begin{array}{l}4.61 \pm \\
1.41\end{array}$ & $\begin{array}{l}21.80 \pm \\
3.70\end{array}$ & $\begin{array}{l}2.68 \pm \\
0.54\end{array}$ & $\begin{array}{l}1.49 \pm \\
0.42\end{array}$ \\
\hline R9 & $\begin{array}{l}83.10 \pm \\
4.63\end{array}$ & $\begin{array}{l}19.10 \pm \\
3.40\end{array}$ & $\begin{array}{l}102.20 \pm \\
5.89\end{array}$ & $\begin{array}{l}9.49 \pm \\
2.43\end{array}$ & $\begin{array}{l}6.09 \pm \\
1.71\end{array}$ & $\begin{array}{l}22.20 \pm \\
1.64 *\end{array}$ & $\begin{array}{l}2.78 \pm \\
0.35\end{array}$ & $\begin{array}{l}1.41 \pm \\
0.22\end{array}$ \\
\hline R10 & $\begin{array}{l}93.28 \pm \\
9.13 *\end{array}$ & $\begin{array}{l}35.56 \pm \\
5.82 * *\end{array}$ & $\begin{array}{l}128.84 \pm \\
14.02 * *\end{array}$ & $\begin{array}{l}10.65 \pm \\
1.43\end{array}$ & $\begin{array}{l}5.50 \pm \\
0.94\end{array}$ & $\begin{array}{l}20.80 \pm \\
2.39\end{array}$ & $\begin{array}{l}3.37 \pm \\
0.55\end{array}$ & $\begin{array}{l}1.62 \pm \\
0.29\end{array}$ \\
\hline R11 & $\begin{array}{l}86.16 \pm \\
6.24\end{array}$ & $\begin{array}{l}26.14 \pm \\
1.89\end{array}$ & $\begin{array}{l}112.30 \pm \\
7.02 *\end{array}$ & $\begin{array}{l}12.06 \pm \\
2.20 * *\end{array}$ & $\begin{array}{l}6.98 \pm \\
0.02 * *\end{array}$ & $\begin{array}{l}21.00 \pm \\
1.41\end{array}$ & $\begin{array}{l}2.50 \pm \\
0.87\end{array}$ & $\begin{array}{l}1.38 \pm \\
0.36\end{array}$ \\
\hline
\end{tabular}

C1- control uninoculated, C2- Penicillium chrysogenum Thom., C3- Bacterial isolate PB 5, R1-R11 -Rhizobial isolates $(\mathrm{p}>0.05 *, \mathrm{p}>0.01 * *)$ 
Table 4 Effects of dual inoculation of PC and Rhizobium on growth of Acacia nilotica

\begin{tabular}{|c|c|c|c|c|c|c|c|c|}
\hline Treatment & $\begin{array}{l}\text { Shoot } \\
\text { height } \\
(\mathrm{cm})\end{array}$ & $\begin{array}{l}\text { Root } \\
\text { length } \\
(\mathrm{cm})\end{array}$ & $\begin{array}{l}\text { Seedling } \\
\text { height } \\
(\mathrm{cm})\end{array}$ & $\begin{array}{l}\text { FBM } \\
\text { leaf } \\
\text { (g) }\end{array}$ & $\begin{array}{l}\text { DBM } \\
\text { leaf } \\
\text { (g) }\end{array}$ & $\begin{array}{l}\text { Collar } \\
\text { dia } \\
(\mathrm{cm})\end{array}$ & $\begin{array}{l}\text { FBM } \\
\text { root } \\
(\mathrm{g})\end{array}$ & $\begin{array}{l}\text { DBM } \\
\text { root } \\
(\mathrm{g}) \\
\end{array}$ \\
\hline $\mathrm{PC}+\mathrm{R} 1$ & $\begin{array}{l}51.04 \pm \\
7.59\end{array}$ & $\begin{array}{l}20.22 \pm \\
1.12\end{array}$ & $\begin{array}{l}71.26 \pm \\
8.46\end{array}$ & $\begin{array}{l}11.96 \pm \\
2.29 * *\end{array}$ & $\begin{array}{l}7.32 \pm \\
1.24 * *\end{array}$ & $\begin{array}{l}21.60 \pm \\
1.67\end{array}$ & $\begin{array}{l}2.97 \pm \\
0.40\end{array}$ & $\begin{array}{l}1.55 \pm \\
0.15\end{array}$ \\
\hline$P C+R 2$ & $\begin{array}{l}89.48 \pm \\
12.31\end{array}$ & $\begin{array}{l}33.82 \pm \\
8.67 * *\end{array}$ & $\begin{array}{l}123.30 \pm \\
8.14 * *\end{array}$ & $\begin{array}{l}11.38 \pm \\
3.04 * *\end{array}$ & $\begin{array}{l}7.76 \pm \\
1.20 * *\end{array}$ & $\begin{array}{l}20.80 \pm \\
1.92\end{array}$ & $\begin{array}{l}2.61 \pm \\
0.25\end{array}$ & $\begin{array}{l}1.46 \pm \\
0.20\end{array}$ \\
\hline$P C+R 3$ & $\begin{array}{l}80.42 \pm \\
12.41\end{array}$ & $\begin{array}{l}33.98 \pm \\
6.84 * *\end{array}$ & $\begin{array}{l}114.40 \pm \\
15.73 * *\end{array}$ & $\begin{array}{l}11.18 \pm \\
1.31 *\end{array}$ & $\begin{array}{l}6.37 \pm \\
0.67 *\end{array}$ & $\begin{array}{l}22.00 \pm \\
2.35\end{array}$ & $\begin{array}{l}2.69 \pm \\
0.26\end{array}$ & $\begin{array}{l}1.59 \pm \\
0.25\end{array}$ \\
\hline $\mathbf{P C}+\mathbf{R 4}$ & $\begin{array}{l}60.04 \pm \\
7.58\end{array}$ & $\begin{array}{l}44.48 \pm \\
2.30 * *\end{array}$ & $\begin{array}{l}104.52 \pm \\
6.94\end{array}$ & $\begin{array}{l}9.30 \pm \\
1.39\end{array}$ & $\begin{array}{l}5.05 \pm \\
0.54\end{array}$ & $\begin{array}{l}23.00 \pm \\
2.24 * *\end{array}$ & $\begin{array}{l}3.32 \pm \\
0.31\end{array}$ & $\begin{array}{l}1.71 \pm \\
0.22\end{array}$ \\
\hline $\mathrm{PC}+\mathrm{R5}$ & $\begin{array}{l}79.40 \pm \\
7.60\end{array}$ & $\begin{array}{l}18.38 \pm \\
1.21\end{array}$ & $\begin{array}{l}97.78 \pm \\
6.53\end{array}$ & $\begin{array}{l}7.91 \pm \\
2.71\end{array}$ & $\begin{array}{l}3.28 \pm \\
1.22\end{array}$ & $\begin{array}{l}22.20 \pm \\
2.59 *\end{array}$ & $\begin{array}{l}2.63 \pm \\
0.68\end{array}$ & $\begin{array}{l}1.46 \pm \\
0.35\end{array}$ \\
\hline $\mathrm{PC}+\mathrm{R6}$ & $\begin{array}{l}82.98 \pm \\
5.42\end{array}$ & $\begin{array}{l}19.04 \pm \\
0.87\end{array}$ & $\begin{array}{l}102.02 \pm \\
5.34\end{array}$ & $\begin{array}{l}10.94 \pm \\
1.32 *\end{array}$ & $\begin{array}{l}5.39 \pm \\
0.79\end{array}$ & $\begin{array}{l}21.60 \pm \\
3.78\end{array}$ & $\begin{array}{l}2.47 \pm \\
0.59\end{array}$ & $\begin{array}{l}1.34 \pm \\
0.47\end{array}$ \\
\hline $\mathbf{P C}+\mathbf{R 7}$ & $\begin{array}{l}59.90 \pm \\
10.03\end{array}$ & $\begin{array}{l}20.26 \pm \\
1.00\end{array}$ & $\begin{array}{l}80.14 \pm \\
9.31\end{array}$ & $\begin{array}{l}9.91 \pm \\
0.78\end{array}$ & $\begin{array}{l}4.97 \pm \\
0.70\end{array}$ & $\begin{array}{l}21.20 \pm \\
1.79\end{array}$ & $\begin{array}{l}2.69 \pm \\
0.61\end{array}$ & $\begin{array}{l}1.64 \pm \\
0.23\end{array}$ \\
\hline $\mathrm{PC}+\mathrm{R8}$ & $\begin{array}{l}80.04 \pm \\
11.14\end{array}$ & $\begin{array}{l}23.72 \pm \\
2.18\end{array}$ & $\begin{array}{l}103.72 \pm \\
9.62\end{array}$ & $\begin{array}{l}10.91 \pm \\
1.03\end{array}$ & $\begin{array}{l}5.89 \pm \\
0.55\end{array}$ & $\begin{array}{l}20.80 \pm \\
2.17\end{array}$ & $\begin{array}{l}2.62 \pm \\
0.60\end{array}$ & $\begin{array}{l}1.31 \pm \\
0.41\end{array}$ \\
\hline $\mathbf{P C}+\mathbf{R 9}$ & $\begin{array}{l}56.26 \pm \\
6.78\end{array}$ & $\begin{array}{l}32.28 \pm \\
2.53 * *\end{array}$ & $\begin{array}{l}88.54 \pm \\
4.41\end{array}$ & $\begin{array}{l}7.13 \pm \\
1.08\end{array}$ & $\begin{array}{l}3.39 \pm \\
10\end{array}$ & $\begin{array}{l}21.20 \pm \\
3.03\end{array}$ & $\begin{array}{l}2.50 \pm \\
0.43\end{array}$ & $\begin{array}{l}1.35 \pm \\
0.27\end{array}$ \\
\hline $\mathbf{P C}+\mathbf{R} 10$ & $\begin{array}{l}92.14 \pm \\
9.92 *\end{array}$ & $\begin{array}{l}32.64 \pm \\
2.02 * *\end{array}$ & $\begin{array}{l}124.78 \pm \\
11.08^{* *}\end{array}$ & $\begin{array}{l}9.72 \pm \\
1.89\end{array}$ & $\begin{array}{l}6.08 \pm \\
0.98\end{array}$ & $\begin{array}{l}21.60 \pm \\
1.34\end{array}$ & $\begin{array}{l}3.84 \pm \\
1.38\end{array}$ & $\begin{array}{l}2.16 \pm \\
0.95\end{array}$ \\
\hline $\mathbf{P C}+\mathbf{R} 11$ & $\begin{array}{l}99.10 \pm \\
6.57 * *\end{array}$ & $\begin{array}{l}31.96 \pm \\
3.80 * *\end{array}$ & $\begin{array}{l}131.06 \pm \\
6.41 * *\end{array}$ & $\begin{array}{l}12.40 \pm \\
1.57 * *\end{array}$ & $\begin{array}{l}6.30 \pm \\
0.12 *\end{array}$ & $\begin{array}{l}21.00 \pm \\
1.00\end{array}$ & $\begin{array}{l}2.68 \pm \\
1.73\end{array}$ & $\begin{array}{l}1.41 \pm \\
0.40\end{array}$ \\
\hline
\end{tabular}

PC-Penicillium chrysogenum Thom. 1, R1-R11 -Rhizobial isolates ( $\mathrm{p}>0.05 *, \mathrm{p}>0.01 * *)$

Table 5 Effects of dual inoculation of PB5 and Rhizobium on growth of Acacia nilotica

\begin{tabular}{|c|c|c|c|c|c|c|c|c|}
\hline Treatment & $\begin{array}{l}\text { Shoot } \\
\text { height } \\
(\mathrm{cm})\end{array}$ & $\begin{array}{l}\text { Root } \\
\text { length } \\
(\mathrm{cm})\end{array}$ & $\begin{array}{l}\text { Seedling } \\
\text { height } \\
(\mathrm{cm})\end{array}$ & $\begin{array}{l}\text { FBM } \\
\text { leaf } \\
\text { (g) }\end{array}$ & $\begin{array}{l}\text { DBM } \\
\text { leaf } \\
(\mathrm{g}) \\
\end{array}$ & $\begin{array}{l}\text { Collar } \\
\text { dia } \\
(\mathrm{cm})\end{array}$ & $\begin{array}{l}\text { FBM } \\
\text { root } \\
(\mathrm{g}) \\
\end{array}$ & $\begin{array}{l}\text { DBM } \\
\text { root } \\
(\mathrm{g}) \\
\end{array}$ \\
\hline PB5 + R1 & $\begin{array}{l}87.56 \pm \\
11.14\end{array}$ & $\begin{array}{l}37.60 \pm \\
7.63 * *\end{array}$ & $\begin{array}{l}125.16 \pm \\
16.69 * *\end{array}$ & $\begin{array}{l}11.84 \pm \\
1.78 * *\end{array}$ & $\begin{array}{l}6.31 \pm \\
0.21 *\end{array}$ & $\begin{array}{l}21.60 \pm \\
1.14\end{array}$ & $\begin{array}{l}2.78 \pm \\
0.30\end{array}$ & $\begin{array}{l}1.63 \pm \\
0.20\end{array}$ \\
\hline $\mathbf{P B 5}+\mathbf{R 2}$ & $\begin{array}{l}91.18 \pm \\
26.56^{*}\end{array}$ & $\begin{array}{l}29.26 \pm \\
5.42 * *\end{array}$ & $\begin{array}{l}120.44 \pm \\
30.17 * *\end{array}$ & $\begin{array}{l}13.02 \pm \\
1.37 * *\end{array}$ & $\begin{array}{l}6.90 \pm \\
1.51^{* *}\end{array}$ & $\begin{array}{l}20.60 \pm \\
1.52\end{array}$ & $\begin{array}{l}2.32 \pm \\
0.52\end{array}$ & $\begin{array}{l}1.07 \pm \\
0.46\end{array}$ \\
\hline PB5 + R3 & $\begin{array}{l}5.66 \pm \\
12.72\end{array}$ & $\begin{array}{l}19.74 \pm \\
1.13\end{array}$ & $\begin{array}{l}75.40 \pm \\
12.66\end{array}$ & $\begin{array}{l}11.08 \pm \\
1.19 *\end{array}$ & $\begin{array}{l}5.87 \pm \\
1.28\end{array}$ & $\begin{array}{l}21.20 \pm \\
0.84\end{array}$ & $\begin{array}{l}2.40 \pm \\
0.46\end{array}$ & $\begin{array}{l}1.41 \pm \\
0.46\end{array}$ \\
\hline PB5 + R4 & $\begin{array}{l}60.68 \pm \\
6.91\end{array}$ & $\begin{array}{l}25.40 \pm \\
3.45\end{array}$ & $\begin{array}{l}86.08 \pm \\
3.51\end{array}$ & $\begin{array}{l}11.51 \pm \\
1.02 * *\end{array}$ & $\begin{array}{l}6.3 \pm \\
0.88^{*}\end{array}$ & $\begin{array}{l}20.80 \pm \\
0.84\end{array}$ & $\begin{array}{l}2.11 \pm \\
0.36\end{array}$ & $\begin{array}{l}1.2 \\
\pm \\
0.22\end{array}$ \\
\hline PB5 + R5 & $\begin{array}{l}111.36 \pm \\
9.59 * *\end{array}$ & $\begin{array}{l}30.30 \pm \\
7.07 * *\end{array}$ & $\begin{array}{l}141.66 \pm \\
13.03^{*}\end{array}$ & $\begin{array}{l}12.99 \pm \\
1.73 * *\end{array}$ & $\begin{array}{l}6.80 \pm \\
0.31^{*}\end{array}$ & $\begin{array}{l}21.00 \pm \\
1.22\end{array}$ & $\begin{array}{l}2.74 \pm \\
0.27\end{array}$ & $\begin{array}{l}1.46 \pm \\
0.17\end{array}$ \\
\hline PB5 + R6 & $\begin{array}{l}60.24 \pm \\
15.49\end{array}$ & $\begin{array}{l}20.46 \pm \\
1.62\end{array}$ & $\begin{array}{l}80.70 \pm \\
15.44\end{array}$ & $\begin{array}{l}12.50 \pm \\
1.73 * *\end{array}$ & $\begin{array}{l}5.78 \pm \\
1.28\end{array}$ & $\begin{array}{l}21.20 \pm \\
0.84\end{array}$ & $\begin{array}{l}3.38 \pm \\
0.72\end{array}$ & $\begin{array}{l}1.78 \pm \\
0.40\end{array}$ \\
\hline PB5 + R7 & $\begin{array}{l}65.28 \pm \\
6.54\end{array}$ & $\begin{array}{l}23.80 \pm \\
2.87\end{array}$ & $\begin{array}{l}89.08 \pm \\
8.09\end{array}$ & $\begin{array}{l}11.73 \pm \\
2.45^{* *}\end{array}$ & $\begin{array}{l}7.47 \pm \\
0.36^{* *}\end{array}$ & $\begin{array}{l}22.20 \pm \\
1.48^{*}\end{array}$ & $\begin{array}{l}2.74 \pm \\
0.20\end{array}$ & $\begin{array}{l}1.51 \pm \\
0.14\end{array}$ \\
\hline PB5 + R8 & $\begin{array}{l}64.74 \pm \\
13.13\end{array}$ & $\begin{array}{l}25.06 \pm \\
3.06\end{array}$ & $\begin{array}{l}89.82 \pm \\
13.55\end{array}$ & $\begin{array}{l}11.97 \pm \\
1.87 * *\end{array}$ & $\begin{array}{l}6.11 \pm \\
0.73\end{array}$ & $\begin{array}{l}21.80 \pm \\
2.17\end{array}$ & $\begin{array}{l}2.85 \pm \\
0.23\end{array}$ & $\begin{array}{l}1.63 \pm \\
0.08\end{array}$ \\
\hline PB5 + R9 & $\begin{array}{l}89.34 \pm \\
8.40\end{array}$ & $\begin{array}{l}30.64 \pm \\
4.27 * *\end{array}$ & $\begin{array}{l}119.98 \pm \\
11.00^{* *}\end{array}$ & $\begin{array}{l}10.68 \pm \\
2.68\end{array}$ & $\begin{array}{l}6.61 \pm \\
2.19^{*}\end{array}$ & $\begin{array}{l}22.60 \pm \\
1.67 *\end{array}$ & $\begin{array}{l}2.57 \pm \\
0.13\end{array}$ & $\begin{array}{l}1.42 \pm \\
0.08\end{array}$ \\
\hline PB5 + R10 & $\begin{array}{l}85.86 \pm \\
8.51\end{array}$ & $\begin{array}{l}31.54 \pm \\
2.16^{* *}\end{array}$ & $\begin{array}{l}117.40 \pm \\
9.64 * *\end{array}$ & $\begin{array}{l}11.32 \pm \\
2.37\end{array}$ & $\begin{array}{l}5.72 \pm \\
1.52\end{array}$ & $\begin{array}{l}22.60 \pm \\
1.67 *\end{array}$ & $\begin{array}{l}2.72 \pm \\
0.38\end{array}$ & $\begin{array}{l}1.61 \pm \\
0.29\end{array}$ \\
\hline PB5 + R11 & $\begin{array}{l}101.70 \pm \\
12.19^{* *}\end{array}$ & $\begin{array}{l}35.92 \pm \\
6.31 * *\end{array}$ & $\begin{array}{l}137.62 \pm \\
13.87 * *\end{array}$ & $\begin{array}{l}11.08 \pm \\
2.14\end{array}$ & $\begin{array}{l}6.58 \pm \\
1.34^{*}\end{array}$ & $\begin{array}{l}21.20 \pm \\
1.64\end{array}$ & $\begin{array}{l}3.29 \pm \\
0.42\end{array}$ & $\begin{array}{l}1.82 \pm \\
0.41\end{array}$ \\
\hline
\end{tabular}

PB5 Bacterial isolate, R1-R11 -Rhizobial isolates ( $>0.05 *, p>0.01 * *)$ 
Table 6 Effects of Triple inoculation of Pencillium chrysogenum, PB 5 and Rhizobium on growth of Acacia nilotica

\begin{tabular}{|c|c|c|c|c|c|c|c|c|}
\hline Treatment & $\begin{array}{l}\text { Shoot } \\
\text { height } \\
(\mathrm{cm})\end{array}$ & $\begin{array}{l}\text { Root } \\
\text { length } \\
(\mathrm{cm})\end{array}$ & $\begin{array}{l}\text { Seedling } \\
\text { height } \\
(\mathrm{cm})\end{array}$ & $\begin{array}{l}\text { FBM } \\
\text { leaf } \\
(g) \\
\end{array}$ & $\begin{array}{l}\text { DBM } \\
\text { leaf } \\
(\mathrm{g}) \\
\end{array}$ & $\begin{array}{l}\text { Collar } \\
\text { dia } \\
(\mathrm{cm})\end{array}$ & $\begin{array}{l}\text { FBM } \\
\text { root } \\
(\mathrm{g}) \\
\end{array}$ & $\begin{array}{l}\text { DBM } \\
\text { root } \\
(\mathrm{g}) \\
\end{array}$ \\
\hline CONTROL & $\begin{array}{l}70.94 \pm \\
6.62\end{array}$ & $\begin{array}{l}19.58 \pm \\
1.44\end{array}$ & $\begin{array}{l}90.52 \pm \\
7.44\end{array}$ & $\begin{array}{l}8.14 \pm \\
1.31\end{array}$ & $\begin{array}{l}3.79 \pm \\
0.67\end{array}$ & $\begin{array}{l}18.60 \pm \\
2.70\end{array}$ & $\begin{array}{l}2.75 \pm \\
0.18\end{array}$ & $\begin{array}{l}1.47 \pm \\
0.13\end{array}$ \\
\hline $\begin{array}{l}\text { PC+PB5 + } \\
\text { R1 }\end{array}$ & $\begin{array}{l}100.80 \pm \\
11.17 * *\end{array}$ & $\begin{array}{l}31.64 \pm \\
5.48 * *\end{array}$ & $\begin{array}{l}132.44 \pm \\
14.34 * *\end{array}$ & $\begin{array}{l}10.80 \pm \\
1.21\end{array}$ & $\begin{array}{l}6.06 \pm \\
0.72\end{array}$ & $\begin{array}{l}21.20 \pm \\
1.64\end{array}$ & $\begin{array}{l}3.09 \pm \\
0.35\end{array}$ & $\begin{array}{l}1.68 \pm \\
0.18\end{array}$ \\
\hline $\begin{array}{l}\text { PC + PB5 + } \\
\text { R2 }\end{array}$ & $\begin{array}{l}68.04 \pm \\
7.98\end{array}$ & $\begin{array}{l}25.36 \pm \\
4.23\end{array}$ & $\begin{array}{l}93.40 \pm \\
9.14\end{array}$ & $\begin{array}{l}11.33 \pm \\
1.82\end{array}$ & $\begin{array}{l}5.65 \pm \\
1.40\end{array}$ & $\begin{array}{l}21.60 \pm \\
0.89\end{array}$ & $\begin{array}{l}2.56 \pm \\
0.60\end{array}$ & $\begin{array}{l}1.55 \pm \\
0.43\end{array}$ \\
\hline $\begin{array}{l}\text { PC + PB5 + } \\
\text { R3 }\end{array}$ & $\begin{array}{l}81.40 \pm \\
23.42\end{array}$ & $\begin{array}{l}27.12 \pm \\
5.73\end{array}$ & $\begin{array}{l}108.52 \pm \\
23.97\end{array}$ & $\begin{array}{l}11.34 \pm \\
1.20\end{array}$ & $\begin{array}{l}6.79 \pm \\
0.68^{*}\end{array}$ & $\begin{array}{l}20.40 \pm \\
0.55\end{array}$ & $\begin{array}{l}3.02 \pm \\
0.18\end{array}$ & $\begin{array}{l}1.79 \pm \\
0.20\end{array}$ \\
\hline $\begin{array}{l}\text { PC + PB5 + } \\
\text { R4 }\end{array}$ & $\begin{array}{l}89.96 \pm \\
10.45\end{array}$ & $\begin{array}{l}21.96 \pm \\
1.73\end{array}$ & $\begin{array}{l}111.92 \pm \\
9.48 *\end{array}$ & $\begin{array}{l}11.01 \pm \\
1.60\end{array}$ & $\begin{array}{l}6.37 \pm \\
0.27^{*}\end{array}$ & $\begin{array}{l}21.20 \pm \\
2039\end{array}$ & $\begin{array}{l}2.78 \pm \\
0.29\end{array}$ & $\begin{array}{l}1.44 \pm \\
0.12\end{array}$ \\
\hline $\begin{array}{l}\text { PC + PB5+ } \\
\text { R5 }\end{array}$ & $\begin{array}{l}98.64 \pm \\
10.25 * *\end{array}$ & $\begin{array}{l}36.94 \pm \\
3.75\end{array}$ & $\begin{array}{l}135.58 \pm \\
11.99 * *\end{array}$ & $\begin{array}{l}11.97 \pm \\
2.38\end{array}$ & $\begin{array}{l}6.21 \pm \\
1.07\end{array}$ & $\begin{array}{l}21.00 \pm \\
2.55\end{array}$ & $\begin{array}{l}3.81 \pm \\
0.57\end{array}$ & $\begin{array}{l}2.05 \pm \\
0.39\end{array}$ \\
\hline $\begin{array}{l}\text { PC + PB5 + } \\
\text { R6 }\end{array}$ & $\begin{array}{l}75.66 \pm \\
5.62\end{array}$ & $\begin{array}{l}32.04 \pm \\
2.05 * *\end{array}$ & $\begin{array}{l}107.70 \pm \\
6.73\end{array}$ & $\begin{array}{l}10.92 \pm \\
1.82\end{array}$ & $\begin{array}{l}6.26 \pm \\
0.64 *\end{array}$ & $\begin{array}{l}21.60 \pm \\
2.41\end{array}$ & $\begin{array}{l}3.02 \pm \\
0.78\end{array}$ & $\begin{array}{l}1.81 \pm \\
0.37\end{array}$ \\
\hline $\begin{array}{l}\text { PC + PB5 + } \\
\text { R7 }\end{array}$ & $\begin{array}{l}90.64 \pm \\
12.61 * *\end{array}$ & $\begin{array}{l}32.22 \pm \\
3.64 * *\end{array}$ & $\begin{array}{l}122.86 \pm \\
12.45^{* *}\end{array}$ & $\begin{array}{l}11.27 \pm \\
2.33\end{array}$ & $\begin{array}{l}7.20 \pm \\
1.12 * *\end{array}$ & $\begin{array}{l}23.60 \pm \\
0.55^{* *}\end{array}$ & $\begin{array}{l}2.96 \pm \\
0.37\end{array}$ & $\begin{array}{l}1.63 \pm \\
0.15\end{array}$ \\
\hline $\begin{array}{l}\text { PC+ PB5+ } \\
\text { R8 }\end{array}$ & $\begin{array}{l}78.60 \pm \\
13.66\end{array}$ & $\begin{array}{l}31.40 \pm \\
2.76^{* *}\end{array}$ & $\begin{array}{l}110.00 \pm \\
11.23\end{array}$ & $\begin{array}{l}11.88 \pm \\
1.35\end{array}$ & $\begin{array}{l}6.70 \pm \\
0.38^{*}\end{array}$ & $\begin{array}{l}22.20 \pm \\
1.79 *\end{array}$ & $\begin{array}{l}2.64 \pm \\
0.48\end{array}$ & $\begin{array}{l}1.39 \pm \\
0.26\end{array}$ \\
\hline $\begin{array}{l}\mathrm{PC}+\mathrm{PB} 5+ \\
\text { R9 }\end{array}$ & $\begin{array}{l}75.56 \pm \\
10.08\end{array}$ & $\begin{array}{l}37.08 \pm \\
3.42 * *\end{array}$ & $\begin{array}{l}112.64 \pm \\
8.00 *\end{array}$ & $\begin{array}{l}11.59 \pm \\
2.16\end{array}$ & $\begin{array}{l}6.78 \pm \\
1.31 *\end{array}$ & $\begin{array}{l}20.80 \pm \\
1.92\end{array}$ & $\begin{array}{l}2.74 \pm \\
0.58\end{array}$ & $\begin{array}{l}1.46 \pm \\
0.33\end{array}$ \\
\hline $\begin{array}{l}\text { PC }+ \text { PB5 + } \\
\text { R10 }\end{array}$ & $\begin{array}{l}83.80 \pm \\
5.77\end{array}$ & $\begin{array}{l}42.06 \pm \\
1.93 * *\end{array}$ & $\begin{array}{l}125.86 \pm \\
4.31 * *\end{array}$ & $\begin{array}{l}13.44 \pm \\
2.45\end{array}$ & $\begin{array}{l}6.96 \pm \\
1.96^{* * *}\end{array}$ & $\begin{array}{l}21.60 \pm \\
2.88\end{array}$ & $\begin{array}{l}2.96 \pm \\
0.50\end{array}$ & $\begin{array}{l}1.61 \pm \\
0.18\end{array}$ \\
\hline $\begin{array}{l}\text { PC + PB5+ } \\
\text { R11 }\end{array}$ & $\begin{array}{l}73.40 \pm \\
6.02\end{array}$ & $\begin{array}{l}40.00 \pm \\
6.64 * *\end{array}$ & $\begin{array}{l}123.40 \pm \\
3.81 * *\end{array}$ & $\begin{array}{l}13.85 \pm \\
1.11\end{array}$ & $\begin{array}{l}7.69 \pm \\
0.76^{* *}\end{array}$ & $\begin{array}{l}23.60 \pm \\
1.52 * *\end{array}$ & $\begin{array}{l}3.28 \pm \\
0.48\end{array}$ & $\begin{array}{l}1.72 \pm \\
0.28\end{array}$ \\
\hline
\end{tabular}

C- control un- inoculated, PC- Penicillium chrysogenum Thom. 1, PB5- Bacterial isolate, R1-R11 -Rhizobial isolates $(\mathrm{p}>0.05 *, \mathrm{p}>0.01 * *)$

\section{Discussion}

The morphological studies done on the Acacia nilotica grown under bio-inoculations elucidated the positive impact of microbial cultures on plant growth, provided suitability of some inoculants for producing better growth, dry biomass in test plants when compared with the uninoculated control. Specifically, the fungal sp. appeared better in providing benefits to these plants in general. It is evident that after inoculation, the increment of seedling height of inoculated seedling is very high as compared to control. The superiority of $P$. chrysogenum is clearly evident indicated the differential response of microbial inoculations towards the growth and development of the host species

Test plants of Acacia nilotica, was inoculated by the most effective mineral solubilisers showed double symbiotic benefits in terms of nitrogen and phosphorus under periodic experimental conditions. Microbial inoculation of tree legume species with mineral solubilisers, nitrogen fixing bacteria not only enhanced the above ground biomass but also provided a well-developed root system which indirectly helpful in balanced nutrient supply. Hence application of such inoculants at nursery stage may produce quality planting material and useful for reforestation programme. In the present study, nodulation pattern in A. nilotica was very poor. Since, study was carried out for only four months; role of inoculated Rhizobium, their effects on performance of host plants is difficult to be interpreted. However, during screening experiments for evaluation of bioinoculants of mineral solubilisers, especially phosphate solubilising microbes, and Rhizobium isolates, the seedlings was found to be manifested with root nodule though it was very poor, could not be recorded for its number, size, and the structure. Hence, the role of Rhizobium inoculants in the performance of test plants cannot be interpreted well. 


\section{Conclusion}

Many of the tropical leguminous trees are reported to fix atmospheric nitrogen through nodule development, endowed with arbuscular mycorrrhizal and other microbial associations. Plantation of such species enrich poor quality soils. Therefore, the seedlings fortified with microbial inoculations may lead to successful in establishment of plants in poor quality sites (Sah et al. 1998, Sahgal et al. 2004). Our study on bioinoculation, selection of PF3, B5, R 11 either individually or in combination may be good proposition for the development of bioinoculant for the development of quality planting material of this forest tree species.

\section{Acknowledgements}

We are thankful to Ministry of environment, forest, climate change, Govt. of India for financial support through project no 23/22/2006-RE Authors are also thankful to the Chief Executive, Regional Plant Resource Centre, Bhubaneswar Odisha, for providing necessary laboratory and field facilities.

\section{References}

Al-Garni SMS. 2006 - Increased heavy metal tolerance of cowpea plants by dual inoculation of an arbuscular mycorrhizal fungi and nitrogen-fixer Rhizobium bacterium. Afr. J. Biotechnol. 5 (2): 133-142

Dabas P, Kaushik JC. 1998 - Influence of Glomus mosseae, phosphorus drought stress on the growth of Acacia niloticaDalbargia sissoo seedlings Ann Biol 14 (1): 91-94

Dash S, Mohapatra AK, Gupta N. 2013 - Growth response of Dalbergia sissoo Roxb to mineral solubilizing bacteria fungi in nursery conditions J of Topical Ecology, 54(1):109-115

Faye J, Plenchette C, Faye A, Peltier R, Lesueur D. 2008 - Symbiotic status of two protected forests of Acacia nilotica vartomentosa in the Senegal river vally Arid L Manag 22: 255-272

Gupta N, Sabat J, Parida R, Kerkatta P. 2007 - Solubilization of tricalcium phosphate rock phosphate by microbes isolated from chromite, iron manganese mine soils Acta botanica croatica (Croatia), 66(2): 197-204.

Hameeda B, Harini G, Rupela OP, Wani SP, Reddy G. 2008 - Growth promotion of maize by phosphate-solubilizing bacteria isolated from composts macrofauna Microbiol Res 163 (2): 234-242

Huda SMS, Sujauddin M, Shafinat S, Uddin MS. 2007 - Effects of phosphorus potassium addition on growth nodulation of Dalbergia sissoo in the nurseryJ For Res 18 (4): 279-282

Purohit U, Mehar SK, Sundarmoorthy S. 2007 - Ecology of soil fungi in Acacia nilotica based agroforestry systems of Rajasthan, IndiaJ Ind Bot Soc 86 (1 \& 2): 86-94

Sah SP, Dutta IC, Haque MS. 1998 - Nursery and field response of sissoo plants (Dalbergia sissoo) to Rhizobium inoculation. Sil. Fenn. 32 (3): 253-259.

Sahgal M, Sharma A, Johri BN, Prakash A. 2004 - Selection of growth promontory Rhizobia for Dalbergia sissoo from diverse soil ecosystems of India Symbiosis 36 (1): 83-96

Sarr A, Lesueur D. 2007 - Influence of soil fertility on the rhizobial competitiveness for nodulation of Acacia senegal Acacia niloticaprovenances in nursery field conditions Wor J Microbiol Biotechnol 23 (5): 705-711

Sharma MP, Bhatia NP, Adholeya A. 2001 - Mycorrhizal dependency growth responses of Acacia niloticaAlbizzia lebbeck to inoculation by indigenous AM fungi as influenced by available soil P levels in a semi-arid Alfisol waste New for 21 (1): 89-104

Sokal RR, Rohlf FJ. 1981 - Biometry: The Principles and Practice of Statistics in Biological Research (Eds-W. H. Freeman). Second Edition. San Francisco.

Talpur FN, Bhanger MI Sherazi STH. 2008 - Intramuscular fatty acid profile of longissimus dorsi semitendinosus muscle from Pateri goats fed under traditional feeding system of Sindh, Pakistan Meat Sci 80 (3): 819-822 
Tewari P, Saxena AK, Rao OP. 2006 - Effect of sodicity and salinity on seedling growth of two early successional agroforestry tree species. Trop. Ecol. 47 (1): 125-132.

Tilak KVBR, Ranganayaki N, Pal KK, De R et al. 2005 - Diversity of plant growth soil health supporting bacteria Curr Sci 89 (1): 136-150

Varma S, Mathur RS. 1989 - Biocoenotic association between nitrogen-fixing phosphatesolubilizing microorganisms Curr Sci 58 (19): 1099-1100 